\title{
Psychiatric nurses expressed conflicting feelings in the decision to use physical restraints
}

Marangos-Frost S, Wells D. Psychiatric nurses'thoughts and feelings about restraint use: a decision dilemma.J Adv Nurs $2000 \mathrm{Feb} ; 31: 362-9$.

QUESTION: What are psychiatric nurses' thoughts and feelings in patient restraint situations and what issues do they face in their decision to restrain?

Design

Ethnographic qualitative study.

\section{Setting}

30 bed, unlocked psychiatric inpatient ward of a community hospital in Toronto, Ontario, Canada.

\section{Participants}

6 registered nurses (age range $42-56$ y, all women) who had 10-15 years of experience in psychiatric nursing and had attended annual educational programmes on the management of aggressive behaviour. The nurses described their experience with 5 patients (age range $18-56$ y, 3 women) who had a diagnosis of personality disorder or schizophrenia and had been in previous restraint situations.

\section{Methods}

Each of the 6 nurses participated in an ethnographic interview to learn how nurses use language, organise their knowledge, and construct meaning about patient restraint. Interviews were taped and transcribed and interview data were scrutinised to determine themes occurring within and across interviews.

\section{Main findings}

The main study finding was the decisional dilemma presented to the nurses during a restraint situation which involved making a choice between risking harm to the patient, other patients, staff, or themselves by not restraining, or physically restraining the patient. 4 themes emerged about the decisional dilemma: framing of the situation as a potential for imminent harm, the unsuccessful search for alternatives to physical restraint, the conflicted nurse, and the conditions of restraint. Framing of the situation involved assessing the potential for imminent harm based on observed patient behaviours and patients' past behaviours. The unsuccessful search for alternatives involved acknowledging that no other alternatives were available that would protect as effectively as physical restraint. The conflicted nurse theme reflected the nurses' thoughts and feelings concerning the perceived conflict between their sense of duty to protect patients and the feeling that the use of restraints was a failure to protect. The conditions of restraint were characterised by 3 facility specific factors that influenced the decision to use restraints: the composition of the inpatient population (can create disruption among other inpatients), the policy of restrained patients being under constant nursing care (requires extra nursing staff), and attitudes of management and physicians (may be unsupportive).

\section{Conclusions}

Psychiatric nurses were faced with a decisional dilemma in patient restraint situations. Their decision was complicated by the potential for harm, unsuccessful search for alternatives, choosing among equally unwelcome options, and unit policies and professional attitudes.

\section{COMMENTARY}

The study by Marangos-Frost and Wells explores the dilemmas encountered by nurses in patient restraint situations within acute psychiatry. The study highlights the complex range of factors that need to be considered by nurses when working with those who harm themselves or attempt to harm others, primarily as a result of diagnosed mental illness. The nurses interviewed were all experienced in psychiatry and were working in an open acute setting. To this end, the study is grounded in the harsh reality of contemporary psychiatric nursing practice and is relevant to nurses working in similar clinical environments who must often make difficult decisions about restraint. Given the ethnographic nature of the enquiry, direct quotes are used well and provide vivid accounts of the situations in which the nurses interviewed considered restraint as their only option.

It is extremely important to note, however, that this study does not clearly define physical restraint, and readers should be advised that the authors are in fact referring to the use of physical devices (ie, leather straps), a practice that is not universal. Consequently, the decisional dilemma that nurses face may involve different considerations. The discussion would also have benefited from greater consideration of the moral reasoning used within clinical decision making. This is especially relevant given the ethical issues surrounding the use of restraint ${ }^{1}$ and the continuing debate about the application of moral knowledge within professional nursing practice. $^{23}$

The study develops several interesting themes that may prove useful in the further study of restraint use within psychiatry, most notably in relation to the links between organisational structures, operational philosophy, and the incidence of restraint use.

Nadine Schofield, MSc, DipN(Lon), RMN, MIHM NHS Executive North West, Warrington, UK

1 Dimond B. Legal aspects of nursing. London: Prentice Hall, 1990.

2 Sarvimäki A. Aspects of moral knowledge in nursing. Sch Inq Nurs Pract 1995;9:343-53.

3 Wurzbach ME. Comfort and nurses' moral choices. J Adv Nurs 1996;24:260-4.
For correspondence: Ms S Marangos-Frost Inpatient Mental Health Services, Rouge Valley Health System, Centenary Health Centre Site, 2867 Ellesmere Road

Toronto, Ontario MIE 4B9, Canada. Fax +1 4162817428

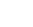

